\title{
$f$-Electron Behavior in Rare Earth Based Systems: Localization or Itinerancy?
}

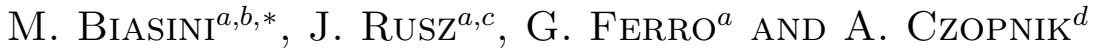 \\ ${ }^{a}$ ENEA, Via Don Fiammelli 2, 40129 Bologna, Italy \\ ${ }^{b}$ Department of Physics, University of California Riverside \\ Riverside CA 92521, USA \\ ${ }^{c}$ Department of Electronic Structures, Faculty of Mathematics and Physics \\ Charles University, Ke Karlovu 5, 12116 Prague 2, Czech Republic \\ ${ }^{d}$ Trzebiatowski Institute of Low Temperature and Structure Research \\ P.O. Box 937, Wrocław, Poland
}

\begin{abstract}
Measurement of the two-dimensional angular correlation of the electronpositron annihilation radiation complemented with $a b$ initio calculations can provide decisive information about the character of the $f$-electrons in rare earth compounds. We provide examples of f-electron localized and f-electron itinerant systems, respectively. (i) In the case of the antiferromagnetic heavy fermion and superconductor $\mathrm{CeIn}_{3}$ the multisheet Fermi surface, reconstructed from our measurements in the paramagnetic phase, agrees closely with the predictions of band structure calculations regarding the Ce $4 f$ electrons as fully localized. (ii) On the other hand, our studies of the antiferromagnet actinide based $\mathrm{UGa}_{3}$ in the paramagnetic phase, compared with calculations which include the effects due to the non-uniform positron density and the electron-positron correlations, produce a substantial evidence that an unconstrained $5 f$-electron itinerant description applies.
\end{abstract}

PACS numbers: 71.18.+y, 71.27.+a, 78.70.Bj

\section{Introduction}

The electronic structure of the intermetallic compounds based on actinides and lanthanides still yields a considerable challenge to our understanding [1]. Thermodynamic and transport properties observed in some $4 f$-based heavy fermion systems have been explained assuming the itinerancy of the $4 f$ electrons. However,

*corresponding author; e-mail: Biasini@physics.ucr.edu 
the interpretation of the de Haas - van Alphen ( $\mathrm{dHvA}$ ) and positron annihilation experiments for several $4 f$ systems (including some heavy fermion) were consistent with the hypothesis of the localization of the $f$ electrons [2]. As a consequence, the itinerancy or localization of the $4 f$ electrons in heavy fermion systems is still a strongly debated argument. For the actinides it has generally been accepted that the $f$-electron character is itinerant in the low $Z$ part of the period $(Z \leq 92)$ and localized at higher $Z \mathrm{~s}$ [1]. Nevertheless, the paucity of measurements aimed at measuring directly the Fermi surface (FS) for both classes of materials, particularly for actinide-based systems [1], and contrasting behaviors (see, for example, Refs. [3-5] for $\mathrm{UGa}_{3}$ and USb) urge to pursue the investigation.

In this paper we have chosen two systems which have attracted a considerable attention for the interplay of heavy fermion behavior, magnetic ordering, and anomalous behavior of the resistivity under the application of pressure. In fact, superconductivity (s.c.) is observed in $\mathrm{CeIn}_{3}$ and a drop in the resistivity, possibly precursor of s.c., is detected in $\mathrm{UGa}_{3}[6,4]$. In particular, the fact that superconductivity in the heavy fermion $\mathrm{CeIn}_{3}$ is observed in a narrow interval of pressure, just about the point of disappearance of magnetic order, and not at higher pressures, suggests that these two phenomena are intimately related [6]. However, the role played by the electrons carrying the magnetic moments to the onset of s.c. (direct contribution to the formation of the Cooper pairs or other) has not been clarified. The Sommerfeld coefficient and Néel temperature $T_{\mathrm{N}}$ (at atmospheric pressure) of $\mathrm{CeIn}_{3}$ are $130 \mathrm{~mJ} / \mathrm{K}^{2} \mathrm{~mol}$ and $10.1 \mathrm{~K}$, respectively. At ambient pressure $\mathrm{CeIn}_{3}$ orders in a simple (type II) magnetic structure where the Ce moments are aligned antiferromagnetically in adjacent (111) ferromagnetic planes. The FS of the antiferromagnetic $(\mathrm{AF})$ phase was investigated experimentally via dHvA experiments [1]. The angular dependence of the dHvA frequency branches observed in $\mathrm{CeIn}_{3}$ differed from those observed for the isostructural non $f$-electron compound $\mathrm{LaIn}_{3}$. However, since the results were not compared to a band structure calculation, it was not established whether these differences were due to itinerancy of the $f$ electrons or to the magnetic Brillouin zone (BZ) of $\mathrm{CeIn}_{3}$ (an fcc structure having two Ce atoms per unit cell, unlike the simple cubic BZ of $\left.\mathrm{LaIn}_{3}\right)$.

In this work, we present our investigations on the electronic structures of these compounds via the two-dimensional angular correlation of the electronposition annihilation radiation (2D-ACAR) measurements. In details, by measuring the distribution $N\left(\theta_{x}, \theta_{y}\right)$ of the deviation angles from anticollinearity of the annihilation gamma rays, the experiment determines a 2-dimensional (2D) projection of the 3D electron-positron (ep) momentum density, $\rho^{\mathrm{ep}}(\boldsymbol{p})$ [7]. The standard Lock-Crisp-West (LCW) transformation [8] reinforces the discontinuities of the electron-positron momentum density $\rho^{\mathrm{ep}}(\boldsymbol{p})$ due to the conduction bands by folding $\rho^{\mathrm{ep}}(\boldsymbol{p})$ back onto the first BZ by translation over the appropriate reciprocal lattice vectors $\boldsymbol{G}$. The result of the summation (denoted as LCW density) is [9] 


$$
\rho_{\mathrm{LCW}}^{\mathrm{ep}}(\boldsymbol{k})=\sum_{n} \theta\left(E_{\mathrm{F}}-\epsilon_{\boldsymbol{k}, n}\right) i n t\left|p s i_{\boldsymbol{k}}^{n}(\boldsymbol{r})\right|^{2}|\phi(\boldsymbol{r})|^{2} g(\boldsymbol{r}) \mathrm{d} \boldsymbol{r} .
$$

Here $\phi$ denotes the positron wave function, $\epsilon_{\boldsymbol{k}, n}$ is the energy eigenvalue of the electron from band $n$ with Bloch wave vector $\boldsymbol{k}$ and wave function $\psi_{\boldsymbol{k}}^{n}$. The factor $g(\boldsymbol{r})$ accounts for the ep correlations [10-12]. If the $\boldsymbol{k}, n$ dependence of the overlap integral in Eq. (1) is negligible, $\rho_{\mathrm{LCW}}^{\mathrm{ep}}(\boldsymbol{k})$ is reduced to the occupancy, i.e. the number of occupied states per $\boldsymbol{k}$ point. On the other hand, if the orbital character of the eigenstates of one outer band (valence or conduction) changes noticeably in the BZ, it is essential to include all the factors of Eq. (1) to compare the theory with the experimental LCW density. Note that the 3D LCW transformation is performed after $\rho^{\mathrm{ep}}(\boldsymbol{p})$ is reconstructed tomographically on the base of its $2 \mathrm{D}$ projections. We will show that in the case of $\mathrm{CeIn}_{3}$, where the positron wave function effect turns out to be negligible, we can produce a reconstruction of the 3D multisheet FS. In the case $\mathrm{UGa}_{3}$, the strong positron effect requires the comparison between measured and calculated LCW-density to extract the information on the $5 f$ electrons character.

\section{Results and discussion}

Technical details of the 2D-ACAR experiments, performed in the paramagnetic phase on single crystals of $\mathrm{CeIn}_{3}$ and $\mathrm{UGa}_{3}$, are reported in Refs. [13, 2, 14]. The ab initio calculations adopted the WIEN2k implementation of the full potential linearized augmented plane waves (FLAPW) method [15] within the local density approximation (LDA). The band structure was calculated treating the $f$ electrons ( $4 f$ and $5 f$ for $\mathrm{CeIn}_{3}$ and $\mathrm{UGa}_{3}$, respectively) either as ordinary band electrons ( $f$-band) or as core-like states $(f$-core) $[2,14]$. A strong indication of the localization of the $4 f$ electrons in $\mathrm{CeIn}_{3}$ was provided by comparing 2-dimensional line integrals of the $f$-band and $f$-core occupancies along the [001] direction with the 2D-LCW folded data of the 2D-ACAR measurement for the same projection direction (see Fig. 1 of Ref. [2]). Our conclusion is also consistent with the measurement of the magnetic moment in the ordered phase $\left(\mu=0.65 \mu_{\mathrm{B}}\right)[16]$, which is rather similar to the expectation value of the saturation moment of the $f$ multiplet in a cubic crystal field (for a cubic symmetry, assuming $J=5 / 2$ and the duplet $\Gamma_{7}$ as a ground state, the saturation moment is $\left.\mu=0.71 \mu_{\mathrm{B}}\right)$. Therefore, in the light of these measurements, a simple picture where the $f$ degree of freedom retains a localized, unhybridized character (and consequently an $f$-core model) seems preferable.

The band structure calculated according to the $f$-core recipe shows that only two bands cross the Fermi level. The two resulting FSs consist of an electron-like spheroidal pocket centered at the $R$ points and a hole-like multiply-connected structure surrounding the $X$ points and the $\Gamma$ point. Since, within the $f$-core model, $\mathrm{CeIn}_{3}$ is a compensated metal, the two FSs enclose equal volumes. The 
3D topology of the FS derived from our experiment and predicted by the theory is shown in Fig. 1. The left part (a) shows (in half BZ) a 3D view of the two isodensity surfaces, identified as FSs, selected at the loci of the largest amplitude variations of the 3D LCW density (due to space limitations, the two sheets, labelled by letters $a$ (online color blue) and $d, e$ (online color orange) are shown in the same figure). To select the threshold values of the LCW density we have followed the procedure described in Ref. [17]. In this case two maxima, corresponding to two FS manifolds, were detected. The related Fermi volumes are $(16 \pm 2) \%$ and $(16 \pm 3) \%$ of the BZ for the $R$-centered electron-like FS $(a)$ and the $\Gamma$-centered hole-like FS $(e, d)$, respectively. These values are in good agreement with those obtained by the $f$-core calculation (shown in Fig. 1b), yielding $18 \%$ of the BZ volume for the electron-like and hole-like FSs. The visual agreement between experiment and theory is striking. In particular, note the small necks of the hole-like FS along the [111] direction, indicated by the white arrows, shown in both figures.
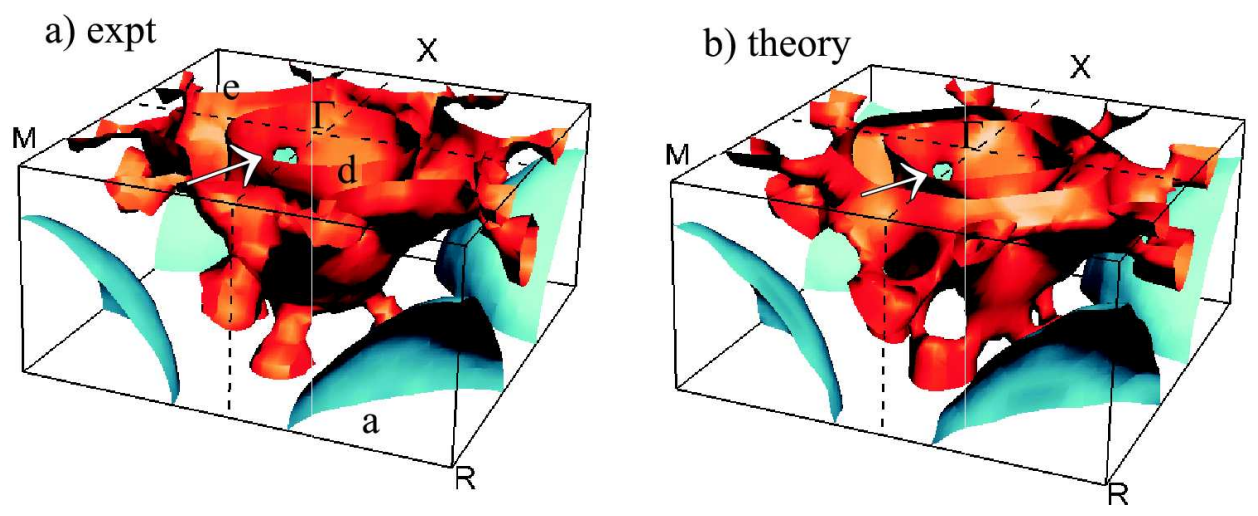

Fig. 1. Expt. (a): The two experimental FSs for $\mathrm{CeIn}_{3}$, shown in half BZ. (i) Hole-like FS: the unoccupied region lies between the inner surface $(d)$, centered at $\Gamma$, and the outer surface (e). (ii) $R$-centered electron-like FS: $(a)$. Theory (b): The two FS sheets predicted by the $f$-core calculation (the same lettering applies). The white arrows address the necks of the hole-like FS in the [111] direction described in the text.

The largest discrepancy between theory and experiment consists in the slightly different topology of the hole-like FS nearby the $X$ points. We have tested further these results by generating from our experimental FSs the angular dependence of the extremal cross-sectional areas expressed as dHvA frequencies (not shown here) [2]. These simulations have confirmed that the $f$-electrons are localized in the paramagnetic phase. On the base of further analysis, described in Ref. [2], we have proposed that the localization of the $f$-electrons persists in the $\mathrm{AF}$ phase, as well. In a way, this conjecture implies that the $f$-electrons should not contribute directly to s.c. Obviously, the question of which electrons participate in the formation of the Cooper pairs is essential to elucidate the s.c. 
mechanism. We propose that a mechanism analogous to the electron-phonon type enhancement in ordinary metals, provided by the electron-magnon interaction, is responsible of the electron pairing that leads to the s.c. In other words, the indirect exchange interaction between the localized $f$ electrons, which is transmitted via the polarization of the conduction electrons (mechanism denoted as RudermanKittel-Kasuya-Yosida (RKKY) interaction) could cause the conduction electron pairing in an analogous manner to that provided by the phonon-conduction electrons interaction in standard BCS superconductors. This possibility is consistent with the narrow region of pressure (around $p_{c}$ ) where s.c. is confined. Indeed, by increasing the pressure above $p_{c}$ the interaction between the $4 f$ electrons should loose its indirect character and therefore the ability to pair the conduction electrons. Therefore, a magnetic interaction appears to be a valid candidate for the pairing of the conduction electrons which arise the s.c. in $\mathrm{CeIn}_{3}$.

The isostructural $\mathrm{CuAu}_{3}$-type $\mathrm{UGa}_{3}$ system is a moderate heavy fermion with the same magnetic structure of $\mathrm{CeIn}_{3}$. The Sommerfeld coefficient and Néel temperature $T_{\mathrm{N}}$ are $52 \mathrm{~mJ} / \mathrm{K}^{2} \mathrm{~mol}$ and $67 \mathrm{~K}$, respectively. Although previous transport and magnetic measurements suggested that the antiferromagnetism of $\mathrm{UGa}_{3}$ is itinerant [18] the dHvA experiments did not confirm this supposition [4].

The LCW $\boldsymbol{k}$-space density of $\mathrm{UGa}_{3}$ showed distinct differences with respect to those of the isostructural $4 f$ electron systems $\mathrm{ErGa}_{3}, \mathrm{TmGa}_{3}$, and $\mathrm{CeIn}_{3}$, where the localization of the $4 f$ electrons was proved by dHvA and 2D-ACAR experiments $[17,19]$. Apart from the different number of $f$-electrons the systems have identical outer electronic configurations. Figure 2 Col. A shows 2D LCW transformations of the experimental projection $\rho^{\mathrm{ep}}\left(p_{x}, p_{y}\right)$, with $p_{z}$ along the [001] axis. It appears that the spectra of the $4 f$ systems (labelled as ErGa $3, \mathrm{TmGa}_{3}, \mathrm{CeIn}_{3}$ in Fig. 2 Col. A) are remarkably similar, consistent with a band structure calculated with the $f$-core recipe $[19,17,2]$. On the other hand, the $\mathrm{UGa}_{3}$ quadrant, apart from the maxima at the MR points, shows trends almost opposite to those displayed in the $4 f$-electron cases. The agreement between LCW densities and $f$-core occupancies for the $4 f$ systems indicates an overall negligible $\boldsymbol{k}$ dependence of the overlap integral in Eq. (1). If instead, an $f$ orbital character is present in the conduction (or valence) electron wave functions of $\mathrm{UGa}_{3}$, a considerable $k$-dependence in the overlap integral of Eq. (1), caused by the different degrees of hybridization along the bands between $f$ orbital degree of freedom and the more delocalized $s, p$ orbitals, could be expected. Therefore, we have compared the alternative descriptions adopted for the $4 f$ electrons of $\mathrm{CeIn}_{3}$ to the case of the $5 f$ of $\mathrm{U}$ ( $5 f$-core, $5 f$-band), including this time the electron-positron matrix elements in the calculation. Moreover, following a semiempirical model where the $5 f$ multiplet is split in itinerant and localized sub-manifolds [20], we have included a third calculation where only two $5 f$-electrons were treated as core states, allowing band $5 f$ states to be occupied (dual- $5 f$ ). 


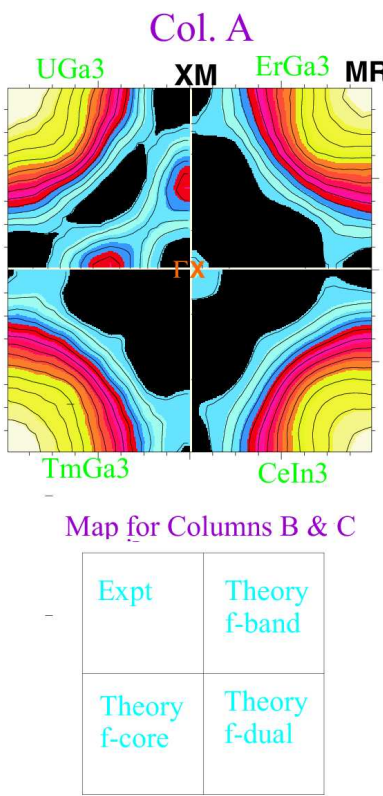

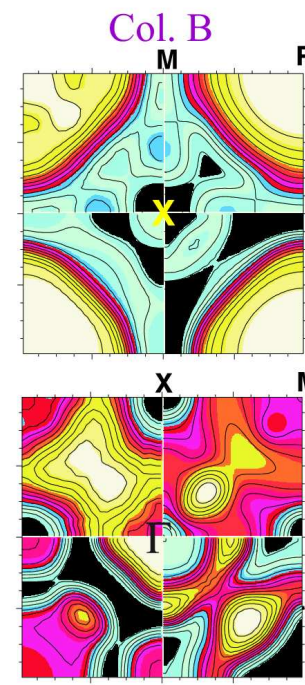

LCW el-pos
Col. C
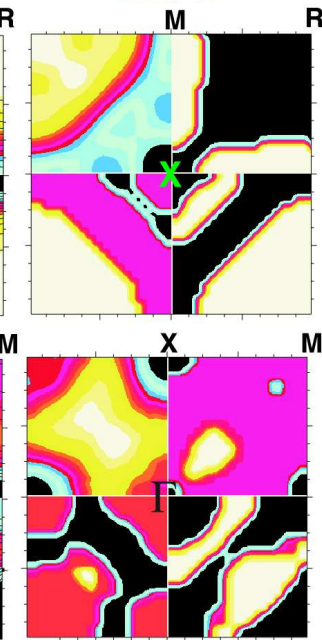

LCW elctrons

Fig. 2. Col. A: $2 \mathrm{D} \rho_{\mathrm{LCW}}^{\mathrm{ep}}\left(k_{x}, k_{y}\right)$ densities of $\mathrm{UGa}_{3}, \mathrm{ErGa}_{3}, \mathrm{TmGa}_{3}$, and $\mathrm{CeIn}_{3}$ obtained from LCW folding the $2 \mathrm{D}$ experimental projection $\rho^{\mathrm{ep}}\left(p_{x}, p_{y}\right)$, with $p_{z}$ along the [001] axis. The contour level spacing (for $\mathrm{UGa}_{3}$ ) corresponds to $0.4 \%$ of the maximum, equivalent to 6 times the statistical uncertainty of the maximum. The labeling describes the projected BZ high symmetry points. Col. B: intersections of the experimental and calculated LCW densities for $\mathrm{UGa}_{3}$ with planes normal to [001] at $k_{z}=0$ (lower) and $k_{z}=\pi / a$ (upper). Calculations were smoothed with a 0.06 a.u. wide $3 \mathrm{D}$ Gaussian function. Panel contents described in the figure map. Col. C: the same as column B with the calculated occupancy replacing $\rho_{\mathrm{LCW}}^{\mathrm{ep} \text { calc }}(\boldsymbol{k})$. The labelling describes the BZ high symmetry points.

The LCW-densities calculated according to these methods ( $5 f$-band, $5 f$-core, dual- $5 f)$, denoted as $\rho_{\mathrm{LCW}}^{\text {ep }}(\boldsymbol{k})$, were then compared to the experimental LCW density. Figure 2 Col. B shows experiment and $5 f$-band, $5 f$-core, and dual-5 $f$ calculations (see figure map) in two high symmetry slices of the BZ (at $k_{z}=0$ (lower) and $k_{z}=\pi / a$ (upper)). Moreover, to appraise the effect of the positron wave function and the electron-positron correlations in $\rho_{\mathrm{LCW}}^{\mathrm{ep} \text { calc }}(\boldsymbol{k})$, Fig. 2 Col. C shows the comparison of the experiment (the same as in Col. C, without contour lines) with the occupancy calculated according to the three methods. Note that the discontinuous changes in gray scale of the calculated occupancy (online color) reflect directly cuts of the FS. It appears that for both slices the $5 f$-band $\rho_{\mathrm{LCW}}^{\mathrm{ep}}(\boldsymbol{k})$ is much more similar to the experiment than the $5 f$-core or dual- $5 f$ calculations. Note, for example, the slice through the $\Gamma$ point (Col. B, lower) where the experiment is practically opposite to $5 f$-core and very different from dual- $5 f$. 
Note also that for the same slice the experiment bears a good resemblance with the $5 f$-band occupancy (Col. C, lower) and therefore with the calculated FS. The agreement between experiment and $\rho_{\mathrm{LCW}}^{\text {epcalc }}(\boldsymbol{k}) 5 f$-band is satisfactory at any slice, taking into account that the comparison is performed on the 3D-reconstructed data, which are obtained on the base of a limited number of projections. However, as one moves away from the $\Gamma$ point along the [001] direction, remarkable differences between occupancy and (calculated) $\rho_{\mathrm{LCW}}^{\mathrm{e} \mathrm{p}_{\text {calc }}}(\boldsymbol{k})$ appear. In particular, at the border of the BZ (upper figure Col. B), only the low at $X$ in $\rho_{\mathrm{LCW}}^{\mathrm{ep} \text { calc }}(\boldsymbol{k})$ (5f-band) reflects the corresponding low in the occupancy. Conversely, the maximum at $R$ in the LCW density contrasts with the corresponding low in the occupancy. In general, the strong positron wave function effects flatten out the large differences of the three band structure calculations. Our calculation shows also that the positron wave function features affect the $k$-dependence of the LCW density much more noticeably than the electron-positron enhancements [10]. We stress that it is only thanks to the powerful 3D analysis that we can draw our propensity for the $5 f$-band model.

In conclusion, we have shown an unprecedented comparison, on a pixel by pixel base, between theory and experiments of the multisheet FS of $\mathrm{CeIn}_{3}$ in the paramagnetic phase. Our simulation of the dHvA signals suggests the persistence of an indirect interaction, RKKY like, between localized $f$ electrons up to the onset of the s.c. transition and hints that the mechanism of pairing has a magnetic origin. Moreover, for the first time we have produced an appreciable direct evidence of $5 f$-electrons itinerancy in $\mathrm{UGa}_{3}$.

We like to thank G. Kontrym-Sznajd, D. Kaczorowski, P.M. Oppeneer, G. Zwicknagl, S. Massidda for stimulating discussions and M. Monge and M. Gemmi for help with software and instrumentation. Moreover, we acknowledge the State Committee for Scientific Research (Poland, grant No 2 P03B 012 25) and the Ministry of Education of the Czech Republic (research program MSM 0021620834).

\section{References}

[1] See, for examples, A.J. Freeman, Physica B 130, 1 (1985); Y. Ōnuki, A. Hasegawa, Handbook on Physics and Chemisrty of Rare Earth, Eds. Karl A. Gschneider Jr., Le Roy Eyring, Vol. 20, North-Holland Publ., Amsterdam 1995, ch. 135 and references therein.

[2] See for example, M. Biasini, G. Ferro, A. Czopnik, Phys. Rev. B 68, 094513 (2003).

[3] A.L. Cornelius, A.J. Arko, J.L. Sarrao, J.D. Thompson, M.F. Hundley, C.H. Booth, N. Harrison, P.N. Oppeneer, Phys. Rev. B 59, 14473 (1999).

[4] D. Aoki, N. Suzuki, K. Miyake, Y. Inada, R. Settai, K. Sugiyama, E. Yamamoto, Y. Haga, Y. Onuki, T. Inoue, K. Kindo, H. Sugawara, H. Sato, H. Yamagami, J. Phys. Soc. Jpn. 70, 538 (2001). 
[5] H. Kumigashira, T. Ito, A. Ashihara, H.-D. Kim, H. Aoki, T. Suzuki, H. Yamagami, T. Takahashi, A. Ochiai, Phys. Rev. B 61, 15707 (2000).

[6] F.M. Grosche, I.R. Walker, S.R. Julian, N.D. Mathur, D.M. Freye, M.J. Steiner, G.G. Lonzarich, J. Phys., Condens. Matter 13, 2845 (2001) and references therein.

[7] S. Berko, in: Proc. Int. School Phys. Enrico Fermi, Eds. W. Brandt, A. Dupasquier, North-Holland Publ. Co., Amsterdam 1983, p. 64.

[8] D.G. Lock, V.H. Crisp, R.N. West, J. Phys. F 3, 561 (1973).

[9] J.H. Kaiser, R.N. West, N. Shiotani, J. Phys. F 16, 1307 (1986).

[10] E. Boronski, R.M. Nieminen, Phys. Rev. B 34, 3820 (1986).

[11] M. Šob, H. Sormann, J. Kuriplach, Adv. Quantum Chem. 42, 77 (2003).

[12] A. Rubaszek, Z. Szotek, W.M. Temmermann, Phys. Rev. B. 65, 125104 (2002).

[13] G. Kontrym-Sznajd, E. Jozefczuk, Mater. Sci. Forum 255-257, 754 (1997).

[14] J. Rusz, M. Biasini, A. Czopnik, Phys. Rev. Lett. 93, 156405 (2004).

[15] P. Blaha, K. Schwartz, J. Luitz, WIEN2k, Tech. Universität Wien, Wien 2001, ISBN 3-9501031-1-2.

[16] J.M. Lawrence, M. Shapiro, Phys. Rev. B 22, 4379 (1980).

[17] M. Biasini, G. Ferro, G. Kontrym-Sznajd, A. Czopnik, Phys. Rev. B 66, 075126 (2002).

[18] D. Kaczorowski, R. Hauser, A. Czopnik, Physica B 230, 35 (1997).

[19] M. Biasini, G. Kontrym-Sznajd, M.A. Monge, M. Gemmi, A. Czopnik, A. Jura, Phys. Rev. Lett. 86, 4612 (2001).

[20] G. Zwicknagl, A.N. Yaresko, P. Fulde, Phys. Rev. B 65, 081103(R) (2003). 